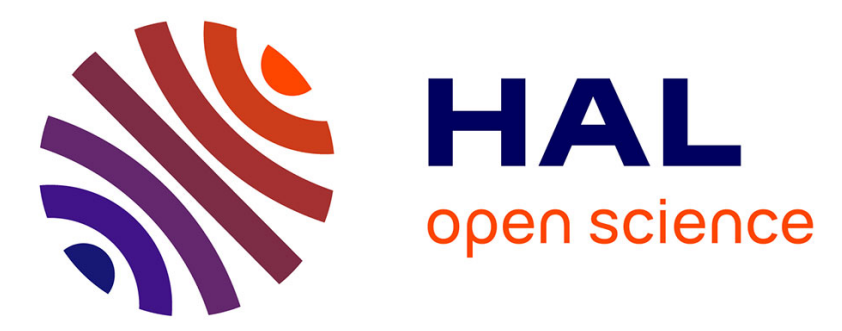

\title{
La problématique du risque dans le financement de projets innovants : une analyse juridique et managériale du crowdfunding base sur le rendement financier
}

\author{
Jean Moussavou, Gurvan Branellec
}

\section{- To cite this version:}

Jean Moussavou, Gurvan Branellec. La problématique du risque dans le financement de projets innovants : une analyse juridique et managériale du crowdfunding base sur le rendement financier. Innovations - Revue d'économie et de management de l'innovation, 2018, Crowdfunding et nouveaux modèles de l'innovation, 56, pp.217-238. 10.3917/inno.pr1.0035 . hal-03523751

\section{HAL Id: hal-03523751 \\ https://hal.science/hal-03523751}

Submitted on 24 Jan 2022

HAL is a multi-disciplinary open access archive for the deposit and dissemination of scientific research documents, whether they are published or not. The documents may come from teaching and research institutions in France or abroad, or from public or private research centers.
L'archive ouverte pluridisciplinaire HAL, est destinée au dépôt et à la diffusion de documents scientifiques de niveau recherche, publiés ou non, émanant des établissements d'enseignement et de recherche français ou étrangers, des laboratoires publics ou privés. 


\title{
LA PROBLEMATIQUE DU RISQUE DANS LE FINANCEMENT DE PROJETS INNOVANTS : UNE ANALYSE JURIDIQUE ET MANAGERIALE DU CROWDFUNDING BASE SUR LE RENDEMENT FINANCIER
}

\author{
Jean MOUSSAVOU \\ Brest Business School- ICI - EA 2652 - IAE/UBO \\ Jean.moussavou@brest-bs.com
}

Gurvan BRANELLEC

Brest Business School - UMR AMURE

Gurvan.branellec@brest-bs.com

Le crowdfunding (CF) s'inscrit aujourd'hui comme une alternative majeure au financement de projets innovants. De multiples entreprises à fort potentiel de croissance sont créées, notamment dans le domaine des technologies digitales, mais pas seulement. Ces créations représentent un potentiel exceptionnel de création de richesse et d'emplois. Pourtant, l'une des difficultés majeures rencontrées par ces entreprises est la réticence des banques à satisfaire à leurs besoins financiers, en raison des incertitudes inhérentes à la viabilité et à la pérennité des projets présentés (échec technique, échec de commercialisation, erreurs de gestion ou de management, etc.). De plus, la collecte de fonds par émission publique d'actions sur les marchés financiers semble problématique, ces entreprises se situant souvent aux premiers stades de leur cycle de vie. De tout ceci découle une exclusion qui s'explique par l'incapacité des outils de financement traditionnels à jouer un rôle efficient dans le financement de cette catégorie d'entreprises. Cette situation a contribué au développement du crowdfunding $(\mathrm{CF})$ ou financement participatif, dont le poids dans le financement de projets innovants ne cesse de croître depuis quelques années (Onnée, 2016). Le CF, quelle que soit la forme retenue, est organisé par des plateformes digitales dont la mission principale est de faciliter l'interaction entre les porteurs de projets, qui cherchent à lever des fonds, et les financeurs potentiels, qui cherchent à s'impliquer dans le financement de ces projets. Dans ses modalités pratiques, le CF est aujourd'hui organisé sous cinq formes : le don (Donation-based crowdfunding), qui s'appuie sur un mécanisme de financement similaire au mécénat; la récompense (y compris le préachat) (Reward-based crowdfunding) qui permet au contributeur d'obtenir une contrepartie non financière (cadeaux, produit prépayé); le prêt (y compris l'emprunt obligataire) (Loan-based crowdfunding ou crowdlending), qui peut être proposé avec ou sans intérêt; la participation au capital (equity-crowdfunding), qui permet au contributeur d'obtenir en retour une part des bénéfices attendus de l'entreprise ; enfin, bien que moins fréquent que les autres modèles, le CF basé sur la redevance (Royalty based crowdfunding), qui permet au contributeur de percevoir une commission basée sur un pourcentage du chiffre d'affaires.

Dans cet article, seules les trois formes de CF basées sur le prêt, l'investissement en capital et la redevance sont retenues. Ces trois formes ont pour objectif d'obtenir un retour financier en contrepartie du soutien apporté, et sont affiliées aux marchés de l'investissement. En cela, elles sont soumises aux cadres de surveillance de la réglementation bancaire et financière.

Aussi, avec la multiplication des fonds levés ${ }^{1}$, le développement du CF a conduit le législateur à mettre en place un cadre juridique propre $^{2}$. La base de ce cadre juridique est claire : pour

\footnotetext{
${ }^{1}$ Selon l'association Financement Participatif France qui réunit des professionnels du crowdfunding, et KPMG, «les moyens alternatifs (financements participatifs, prêts en ligne...) pour financer un projet ont plus que doublé
} 
que le CF devienne une alternative de financement viable et durable, les contributeurs doivent être protégés des risques inhérents à l'activité. Cependant, si le cadre juridique mis en place couvre plusieurs points importants, certains questionnements demeurent : qu'est-ce que le risque en crowdfunding ? Comment ce risque est-il évité, atténué ou empêché dans les plateformes de crowdfunding (PC) ? Et dans quels cas la responsabilité des PC et/ou celle des porteurs de projets peut-elle être engagée?

Pour tenter de répondre à ces questions, notre analyse se positionne à la croisée des domaines des sciences de gestion et du droit, et s'ajoute à la littérature en développement dans le CF, pour laquelle la réglementation financière n'a reçu que très peu d'attention. Or la réglementation apparait omniprésente dans les processus (Quaglia, 2010, p. 3) et le management des plateformes. En cela, son intégration dans les analyses nous semble pertinente. Sur le plan méthodologique, le travail s'appuie sur une démarche qualitative exploratoire à visée compréhensive. L'objectif ici étant de comprendre la portée des dispositifs managériaux et juridiques mis en place pour solutionner, du moins en partie, la problématique du risque dans l'investissement en $\mathrm{CF}$.

Les propos qui suivent sont organisés comme suit : dans une première partie, sera mise en évidence la nature des risques généraux et spécifiques inhérents à l'activité du CF. La deuxième partie présente, après un développement de la méthodologie de recherche adoptée, les actions mises en place pour pallier les risques, avec une double perspective : managériale en précisant comment les PC font face aux risques et la façon dont ces risques sont traités ; juridique avec la règlementation mise en place visant à prévenir la réalisation des risques et, lorsque ceux-ci se réalisent, les mécanismes de responsabilité qui s'appliquent. Enfin, une conclusion développera les principaux apports de ce travail.

\section{LA NATURE DES RISQUES INHERENTS AU CROWDFUNDING BASE SUR LE RENDEMENT FINANCIER (CBRF)}

Dans cette section, nous fournissons un aperçu des risques du CF basé sur le rendement financier (CBRF). L'objectif visé ici étant de mieux cerner les caractéristiques clefs des risques dans ce domaine, qui puissent nous permettre d'explorer les enjeux dans notre étude empirique. Les risques généraux y sont décrits, ainsi que ceux spécifiques liés aux fraudes éventuelles.

\section{Les risques généraux}

Alors que les risques encourus dans les circuits financiers traditionnels sont connus et anticipés pour certains (risque de contrepartie, risque de taux, risque de change, risque d'illiquidité, etc. $)^{3}$, la question du risque en CF reste encore peu abordée dans la littérature, en raison du développement récent de cette activité. Une des premières analyses dans l'Hexagone a été développée par Dardour (2015), qui s'intéresse aux risques tels que perçus par les gestionnaires de plateformes en equity-crowdfunding. L'analyse démontre que les PC

\footnotetext{
en 2016. Le CF à lui seul représente 37\% du total». «Le cabinet Forbes estime que le marché mondial du crowdfunding pèsera 1000 milliards de dollars (dont 7 milliards rien que pour la France) à l'horizon 2020 » (Financement Participatif France, 2017).

${ }^{2}$ L'ordonnance ${ }^{\circ} 2014-559$ du 30 mai 2014 relative au financement participatif, complétée par les dispositions du décret $\mathrm{n}^{\circ} 2014-1053$ du 16 septembre 2014 et celles du décret $n^{\circ} 2016-1453$ du 28 octobre 2016.

${ }^{3}$ Pour un approfondissement sur les risques en finance, $C f$. Vernimmen et al. (2017).
} 
sont des «intermédiaires financiers chargés de collecter l'information en vue de réduire le risque pour l'investisseur ». En outre, la contribution de la communauté d'investisseurs à la sélection et à l'évaluation des risques est prédominante. Pour cet auteur, le traitement du risque suggère ainsi que les PC et les investisseurs s'engagent dans l'évaluation des risques et du potentiel des projets présentés. De fait, si certaines similitudes peuvent exister entre le crowdfunding et les systèmes de financement traditionnels (Business Angels, capital-risque, banques, etc.), une différence concerne le transfert des risques vers la foule (crowd) d'investisseurs-contributeurs. En effet, contrairement aux modèles traditionnels, la PC ne participe pas au financement proprement dit. La PC ne prête pas pour son propre compte, le financement étant effectué par la foule d'internautes, d'où un transfert du risque vers ces derniers. La fonction de la PC se concentre alors sur la rencontre entre les contributeurs et les porteurs de projets en fournissant certaines fonctionnalités, et en fonctionnant comme un marché d'échange électronique afin de surmonter les asymétries d'information et minimiser les coûts de transaction (Mahadevan, 2000).

Aussi, dans une perspective différente, Agrawal et al. (2013) décrivent les risques du CBRF en se référant aux quatre étapes du processus d'investissement ( $C f$ tableau 1). Ils font valoir notamment le manque de due-diligence suffisant des projets, et le manque d'expertise financière des contributeurs.

\section{Tableau 1 - Les risques CBRF}

\begin{tabular}{|c|c|}
\hline Phase & Risques \\
\hline $\begin{array}{l}\text { 1. Sélection et } \\
\text { évaluation } \\
\text { projets }\end{array}$ & $\begin{array}{l}\text { - Absence de vérifications préalables des projets par les investisseurs } \\
\text { en raison d'un faible montant engagé ou du manque d'expertise } \\
\text { financière ; } \\
\text { - Décisions d'investissement biaisées ou trop optimistes (problèmes de } \\
\text { comportements opportunistes); } \\
\text { - Risques liés aux projets et aux asymétries d'information entre les } \\
\text { contributeurs et les porteurs de projets (souvent en raison de la } \\
\text { distance géographique). }\end{array}$ \\
\hline 2. Investissement & $\begin{array}{l}\text { - Risque de divulgation des caractéristiques du projet ; } \\
\text { - Absence de diversification des risques ; } \\
\text { - Risque de comportements mimétiques. }\end{array}$ \\
\hline $\begin{array}{l}\text { 3. Post- } \\
\text { investissement }\end{array}$ & $\begin{array}{l}\text { - Asymétrie d'information entre les contributeurs et les porteurs de } \\
\text { projets ; } \\
\text { - Contrairement aux Business Angels et aux capital-risqueurs, peu ou } \\
\text { pas de relations avec le porteur de projet permettant de vérifier la } \\
\text { conformité sur l'usage réel des fonds (risques de fraudes ou de } \\
\text { mauvaise gouvernance); } \\
\text { - En raison du grand nombre, la gestion des relations avec les } \\
\text { contributeurs est susceptible d'être plus coûteuse (commentaires, } \\
\text { attention, interactions). }\end{array}$ \\
\hline 4. Sortie & $\begin{array}{l}\text { - Illiquidité éventuelle des actifs (difficultés de vendre ou de sortir); } \\
\text { - Possibilités de sortie souvent pensées en tant que stratégie de sortie, } \\
\text { et non en tant que modalités de sortie certaines. }\end{array}$ \\
\hline
\end{tabular}

Source : Agrawal et al. (2013) et compléments des auteurs

Pour leur part, Kirby et Worner (2014, p. 5) classent les risques du CBRF en cinq catégories : le risque de défaut, le risque de fermeture/échec de la plateforme, le risque de fraude ou d'actes illicites, le risque d'illiquidité et, enfin, le risque de cyber-attaque ( $C f$. tableau 2 ). 
Tableau 2 - Les risques du CBRF

\begin{tabular}{|c|c|}
\hline Catégorie de risque & Explications \\
\hline 1. Risque de défaut & $\begin{array}{l}\text { Les plateformes Anaxago et WiSeed ont ainsi été confrontées aux } \\
\text { premiers échecs du crowdfunding sur des projets immobiliers en } \\
\text { France (projets du groupe Terlat) }{ }^{4} \text {. Selon une étude de la Coface } \\
(2015) \text {, le taux de défaillance moyen des jeunes entreprises } \\
\text { innovantes est de } 2,3 \% \text {. }\end{array}$ \\
\hline $\begin{array}{l}\text { 2. Risque de } \\
\text { fermeture ou d'échec } \\
\text { de la plateforme }\end{array}$ & $\begin{array}{l}\text { La plateforme Isodev, spécialisée dans le financement d'entreprises } \\
\text { par l'intermédiaire de prêts participatifs, a par exemple annoncé sa } \\
\text { faillite en 2015. C'est également le cas de MyMajorCompany, } \\
\text { première plateforme apparue en France en 2007, qui a annoncé } \\
\text { l'arrêt de son activité de CF pour se concentrer sur son label musical } \\
\text { participatif. On peut également citer les exemples de FriendsClear en } \\
\text { France et Microcapital aux USA, qui ont cessé leur activité } \\
\text { respectivement en } 2013 \text { et } 2014 \text {. Finalement, pour un contributeur } \\
\text { ayant engagé des fonds via une plateforme en faillite, le risque est de } \\
\text { perdre tout ou partie de son investissement initial. }\end{array}$ \\
\hline $\begin{array}{l}\text { 3. Risque de fraude } \\
\text { et d'actes illicites } \\
\text { (ce point sera } \\
\text { approfondi dans la } \\
\text { section suivante) }\end{array}$ & $\begin{array}{l}\text { Netfinancement.com, une plateforme se présentant comme leader du } \\
\text { prêt participatif en France, proposait ainsi d'investir dans des sociétés } \\
\text { inexistantes ou douteuses, ou dans des projets déjà financés par } \\
\text { d'autres plateformes }{ }^{5} \text {. Aussi, bien qu'aucun cas ne soit avéré, le CF } \\
\text { peut également être vecteur d'opérations illicites (blanchiment } \\
\text { d'argent et financement du terrorisme notamment) car il présente une } \\
\text { certaine opacité créée par l'anonymat même du caractère en ligne de } \\
\text { l'activité (Tracfin, 2016). }\end{array}$ \\
\hline $\begin{array}{l}4 . \\
\text { d'illiquidité }\end{array}$ & $\begin{array}{l}\text { Les investisseurs peuvent avoir des difficultés à vendre leurs } \\
\text { participations. }\end{array}$ \\
\hline $\begin{array}{l}\text { 5. Risque } \\
\text { cyberattaque }\end{array}$ & $\begin{array}{l}\text { La plateforme Kickstarter, basée aux États-Unis, s'était ainsi déclarée } \\
\text { victime d'une intrusion dans ses serveurs en } 2014 \text { en déplorant des } \\
\text { dommages collatéraux sur ses utilisateurs }{ }^{6} \text {. Aussi, même si la } \\
\text { plateforme assurait à l'époque qu'aucune information bancaire } \\
\text { n'avait été dérobée, elle reconnaissait toutefois que des noms } \\
\text { d'utilisateurs, des numéros de téléphone ou encore des adresses mail } \\
\text { et postales ont pu être subtilisés lors de cette acte malveillant.< }\end{array}$ \\
\hline
\end{tabular}

Source : Kirby et Worner (2014) et adaptations des auteurs

Kirby et Worner (2014) expliquent également que le CBRF pourrait poser un risque systémique à long terme compte tenu de sa taille, de sa nature transfrontalière et de la propagation des risques grâce aux pratiques de la titrisation et de l'implication progressive des banques. Premièrement, si la taille actuelle du marché est relativement réduite pour entrainer un risque systémique, le crowdfunding (CF) a le potentiel de devenir un marché plus important en peu de temps, compte tenu de son développement rapide. Deuxièmement, les activités transfrontalières du CF sont nouvelles et nécessitent donc un travail plus approfondi

\footnotetext{
${ }^{4}$ Source :http://bfmbusiness.bfmtv.com/entreprise/un-premier-rate-a-3-millions-d-euros-pour-le-crowdfundingimmobilier-1084207.html.

${ }^{5}$ Source :http://www.capital.fr/finances-perso/crowdfunding/arnaque-crowdfunding-netfinancement-le-site-quipropose-d-investir-dans-des-projets-bidons-1143502.

${ }^{6}$ Source : http://www.itespresso.fr/cyber-attaque-bapteme-feu-kickstarter-72797.html.
} 
pour comprendre les implications juridiques et les complexités transfrontalières. Cette nouveauté pourrait être une source de risque systémique à l'avenir. Troisièmement, la titrisation possible des prêts non garantis du CBRF ouvre le marché à de nouveaux types de produits, mais ouvre également le reste du secteur bancaire et financier à de possibles titrisations de ces prêts, qui sont non garantis par nature. Le danger est donc celui d'une bulle, comme celle de la «nouvelle économie » des années 1999-2000 ou celle du Subprime amorcée en 2007.

Enfin, l'anonymat créé par l'aspect en ligne du CF renforce les probabilités de fraudes dont les caractéristiques ont été mises en évidence par certains auteurs.

\section{Les possibilités de fraudes}

Sur la base d'une enquête, Cornell et Luzar (2014, p. 2-3) fournissent un aperçu de différents cas de menaces de fraudes qui pourraient se présenter dans les campagnes de CF. Ils proposent une taxonomie des situations possibles résumées dans le tableau qui suit (Tableau $3)$.

Tableau 3 - Fraudes et CBRF

\begin{tabular}{|l|l|}
\hline Catégorie de menace & Explications \\
\hline 1. Fraude anticipée & $\begin{array}{l}\text { Une campagne de CF suspecte peut-être clôturée par la plateforme avant } \\
\text { que les fonds engagés ne soient versés au porteur de projet. }\end{array}$ \\
\hline 2. Fraude avortée & $\begin{array}{l}\text { Elle se produit lorsqu'une campagne de collecte est rejetée sommairement } \\
\text { par la plateforme. Alors que les projets peuvent ne pas être retenus pour } \\
\text { une variété de raisons, allant d'une simple incomplétude du dossier à des } \\
\text { erreurs techniques, il y a certainement des projets qui sont écartés après un } \\
\text { filtrage par la plateforme parce qu'ils comportent un risque de fraude. }\end{array}$ \\
\hline 3. Tentative de fraude & $\begin{array}{l}\text { Elle pourrait se produire lorsqu'un porteur de projet utilise un droit de } \\
\text { propriété intellectuelle dont il n'est pas propriétaire. Le porteur de projet } \\
\text { peut lui-même être victime de contrefaçon, notamment de la part } \\
\text { d'entreprises établies ou d'autres individus, qui peuvent utiliser leurs } \\
\text { possibilités financières immédiates pour tirer parti des idées circulant sur } \\
\text { les plateformes. }\end{array}$ \\
\hline 4. Fraude perçue & $\begin{array}{l}\text { Liée aux accusations de fraudes pouvant survenir lorsque les contreparties } \\
\text { (récompenses, retour sur investissement, etc.) sont considérablement } \\
\text { retardées. }\end{array}$ \\
\hline $\begin{array}{l}\text { 5. Fraude du créateur- } \\
\text { contributeur }\end{array}$ & $\begin{array}{l}\text { Les porteurs de projets peuvent contribuer à leurs propres campagnes, soit } \\
\text { directement, soit par l'intermédiaire de substituts dans la seule perspective } \\
\text { de créer une dynamique susceptible d'induire en erreur les contributeurs } \\
\text { potentiels. }\end{array}$ \\
\hline $\begin{array}{l}\text { 6. Fraude de } \\
\text { plateforme }\end{array}$ & $\begin{array}{l}\text { Liée à l'émergence de nombreuses plateformes de niche. Or plus le nombre } \\
\text { de plateformes est important, plus les créateurs de PC eux-mêmes risquent } \\
\text { de se livrer à des fraudes ou permettre les fraudes (Cf. Cas Netfinancement } \\
\text { cité plus haut). }\end{array}$ \\
\hline \multicolumn{2}{|c|}{ Source : Cornell et Luzar (2014, p. 2-3) et compléments des auteurs } \\
\hline
\end{tabular}

En définitive, le CF nous semble accentuer le niveau des risques, en même temps qu'il en ajoute de nouveaux à ceux déjà présents dans les circuits de financement traditionnels, en raison notamment des caractéristiques d'anonymat et de la dématérialisation des échanges entre les contributeurs et les porteurs de projet. Cette situation conduit à nous interroger sur les dispositifs managériaux et juridiques mis en place pour pallier les risques (au sens large : risques généraux et risques de fraudes). 


\section{LES DISPOSITIFS JURIDIQUES ET MANAGERIAUX DE PREVENTION ET DE REDUCTION DES RISQUES}

Ces dispositifs concernent d'une part, les dispositions réglementaires initiées par le législateur pour prévenir la réalisation des risques et sécuriser les pratiques du CF et, d'autre part, les mécanismes mis en place par les PC dans le processus de sélection des projets. Les propos qui suivent présentent tout d'abord la méthodologie de recherche adoptée, puis les résultats observés seront présentés.

\section{Méthodologie de recherche : de l'approche documentaire à la méthode de cas par comparaisons intersites}

L'approche méthodologique a été structurée en deux étapes. Tout d'abord, pour appréhender les modalités de gestion des risques par les PC, nous avons choisi d'utiliser la méthodologie d'étude de cas par comparaisons inter-sites (ou étude de cas multiples) telle que définie par Eisenhardt (1989) et Yin (1994). Il s'agit d'une approche basée sur une analyse de plusieurs cas simultanément. L'approche est intéressante ici en ce qu'elle invite à l'ouverture et ne restreint pas l'analyse à un cadre limité de travail. Elle incite plutôt à enrichir l'observation du terrain et permet d'identifier ce qui peut distinguer un cas d'un autre ou découvrir des régularités entre les cas étudiés. Pour cela, nous nous sommes appuyés sur l'observation d'un échantillon de PC parmi celles régulées par les autorités françaises, et répertoriées par 1'association Financement Participatif France (Financement participatif France (FPF), 2017) ${ }^{7}$. Dans ce cadre, nous avons procédé à l'analyse des informations contenues sur les sites internet desdites PC (avertissements sur les risques, processus de sélection des projets, suivi et reporting, possibilités de sorties envisagées, conditions d'utilisations, etc.). Nous avons également collecté des données primaires à partir d'une série d'entretiens semi-directifs réalisés avec certaines PC de l'échantillon (5 plateformes: Anaxago, GwenneG, SmartAngels, Sowefund, Wissed). Le guide d'entretien était structuré autour de plusieurs points, dont la vision générale des répondants sur le crowdfunding, le rôle des acteurs, les enjeux et les pratiques dans le traitement des risques pendant la levée de fonds, et le suivi des projets après la levée de fonds. Les entretiens ont durée en moyenne $1 \mathrm{~h}$ et il a été possible de les enregistrer. La redondance dans l'information recueillie a été pour nous un signe de saturation. En effet, lors des 2 derniers entretiens, il n'y avait plus de nouvelles données pertinentes et les réponses recoupaient tous les entretiens précédents.

Par ailleurs, le choix des PC de l'échantillon s'est basé sur la possibilité de recueillir une information suffisamment large sur les sites internet des plateformes, et en lien avec notre problématique de recherche. En plus de veiller à la richesse de l'information, nous avons essayé de choisir un échantillon susceptible de répliquer en miniature la population de base (Fortin, 1996). En d'autres termes, nous avons essayé de choisir un échantillon de telle sorte qu'on puisse y retrouver les caractéristiques hétérogènes qui distinguent les différentes PC sur le terrain. C'est ce qui explique le choix définitif de 13 plateformes retenues dans notre échantillon ( $C f$. tableau 4), sur une population de base de 68 plateformes membres de l'Association Financement Participatif France au 05/06/2017. Aussi, concernant toujours l'échantillon, nous pensons que la différence entre la population de base et le nombre de plateformes de l'échantillon ne compromet en rien nos analyses. En effet, d'après Fortin (1996), dans une méthodologie d'étude de cas, le chercheur doit plutôt se soucier d'identifier

\footnotetext{
${ }^{7}$ http://financeparticipative.org/
} 
dans quelle mesure les cas sélectionnés sont susceptibles de fournir des données valides et complètes plutôt que de s'interroger sur leur représentativité. Enfin, concernant la méthodologie d'analyse de données, la nature qualitative des informations collectées nous a conduits à opter pour une «analyse de contenu ». Aussi, s'agissant de données issues de cas multiples, l'analyse repose sur les recommandations de Miles et Huberman (2003). Conformément aux préconisations de ces auteurs, les données recueillies ont d'abord fait l'objet d'une analyse intra-site. Les informations recueillies à la suite de ce premier traitement ont alors été comparées d'un cas à l'autre afin de dégager des régularités, ou des points de convergence et de différence. Le but était de faire ressortir des éléments d'observations qui soient justifiables au regard de notre problématique de recherche.

Tableau 4 - Liste des PC de l'échantillon

\begin{tabular}{|c|c|c|c|}
\hline Plateformes & $\begin{array}{l}\text { Type de } \\
\text { financement } \\
*\end{array}$ & $\begin{array}{l}\text { Statut } \\
* *\end{array}$ & $\begin{array}{l}\begin{array}{l}\text { Positionnement sectoriel, géographique et/ou } \\
\text { particularités }\end{array} \\
\end{array}$ \\
\hline 1001Pact & I et $\mathrm{D}$ & CIP & $\begin{array}{l}\text { Plateforme dédiée au financement de l'entrepreneuriat } \\
\text { social }\end{array}$ \\
\hline Afrikwity & I & CIP & $\begin{array}{l}\text { Plateforme dédiée au financement de start-up ou PME } \\
\text { innovantes en l'Afrique. }\end{array}$ \\
\hline Anaxago & I et $\mathrm{D}$ & CIP & $\begin{array}{l}\text { Plateforme permettant de rapprocher le financement } \\
\text { des entreprises innovantes et les investisseurs } \\
\text { particuliers. }\end{array}$ \\
\hline Enerfip & I et $\mathrm{D}$ & $\begin{array}{l}\text { IFP et } \\
\text { CIP }\end{array}$ & $\begin{array}{l}\text { Plateforme destinée à financer des projets d'énergies } \\
\text { renouvelables et de transition énergétique. }\end{array}$ \\
\hline Feedelios & I et $\mathrm{D}$ & CIP & $\begin{array}{l}\text { Plateforme destinée aux sociétés dont le siège social } \\
\text { est basé en DOM-TOM. }\end{array}$ \\
\hline GwenneG & I et $\mathrm{D}$ & $\begin{array}{l}\text { IFP et } \\
\text { CIP }\end{array}$ & $\begin{array}{l}\text { Plateforme ancrée sur le territoire breton et soucieuse } \\
\text { de soutenir l'économie régionale. }\end{array}$ \\
\hline Investbook & $\mathrm{D}$ & CIP & $\begin{array}{l}\text { Plateforme dédiée à l'investissement d'entreprises par } \\
\text { souscription d'obligations. }\end{array}$ \\
\hline $\begin{array}{l}\text { MyPharmaCom } \\
\text { pany }\end{array}$ & I & IFP & Plateforme dédiée au financement liés à la santé. \\
\hline Smartangels & I & CIP & $\begin{array}{l}\text { Plateforme française exclusivement dédiée à } \\
\text { l'investissement dans les start-ups et PME de } \\
\text { croissance. }\end{array}$ \\
\hline Sowefund & I & CIP & $\begin{array}{l}\text { Plateforme qui rassemble les professionnels du } \\
\text { financement (Business angels, etc.) et les particuliers. }\end{array}$ \\
\hline TheGoodInvest & I & CIP & $\begin{array}{l}\text { Plateforme spécialisée dans les univers de l'hôtellerie, } \\
\text { la restauration et le tourisme }\end{array}$ \\
\hline WeDoGood & $\mathrm{I}$ & IFP & Première plateforme de financement en royalties \\
\hline$\underline{\text { Wiseed }}$ & D et I & PSI & $\begin{array}{l}\text { Plateforme permettant d'investir dans le capital de } \\
\text { startups et PME, mais également de contribuer au } \\
\text { financement de programmes immobiliers. }\end{array}$ \\
\hline \multicolumn{4}{|c|}{$\begin{array}{l}* \text { I : Investissement (Capital, Royalties) } \\
D: \text { Dettes (prêts, obligations, minibons) }\end{array}$} \\
\hline
\end{tabular}

Les analyses intersites tirées de l'étude empirique ont permis de dégager les observations présentées dans les sections qui suivent.

Ensuite, une analyse documentaire a été conduite pour collecter les données juridiques ayant permis d'appréhender les dispositions règlementaires de prévention des risques. Dans ce 
cadre, nous avons analysé le Code monétaire et financier réglementant les acteurs du secteur bancaire et financier, ainsi que certaines dispositions du Code civil liés au traitement de la responsabilité contractuelle. De façon plus spécifique différents textes ont également alimenté nos analyses (ils sont cités de manière chronologique) : les dispositions de la loi pour la confiance en l'Économie numérique $\mathrm{n}^{\circ} 2004-575$ du 21 juin 2004, la loi Hamon n²014-344 du 17 mars 2014 sur la consommation, l'ordonnance $n^{\circ} 2014-559$ du 30 mai 2014 relative au financement participatif, complétée par les dispositions du décret n²014-1053 du 16 septembre 2014, l'Ordonnance $\mathrm{n}^{\circ}$ 2016-131 du 10 février 2016 portant sur le droit des contrats et enfin le décret $\mathrm{n}^{\circ} 2016-1453$ du 28 octobre 2016 relatif aux titres et aux prêts proposés dans le cadre du financement participatif.

\section{Les dispositifs de réduction des risques au sein des plateformes}

Les porteurs de projets proposant une idée en vue d'un financement ont à fournir des documents d'offre préparés par eux-mêmes. Bien que les documents demandés puissent être sensiblement divergents d'une PC à une autre, ils suivent une composition relativement semblable : présentation du porteur de projet et/ou de l'équipe de gestion; présentation du modèle d'affaires et des projections de ventes ; présentation des produits et/ou services proposés ; de l'action commerciale projetée ; de la cohérence financière ; de la propriété intellectuelle et, enfin, de l'analyse du marché et/ou du secteur d'activité (concurrence, clientèle visée, etc.). Aussi, au-delà des objectifs liés à l'évaluation des risques intrinsèques au projet, certaines informations collectées permettent de vérifier l'identité, les motivations et les antécédents du porteur de projet pour éviter notamment tout risque de fraude. Les projets sont ainsi généralement filtrés avant d'être proposés aux contributeurs potentiels sur le site internet de la plateforme. Certaines plateformes proposent des rencontres physiques entre les porteurs de projets et les contributeurs lors de réunions d'informations collectives. Ce type de rencontres permet de collecter une information plus claire sur le projet, mais aussi sur la personnalité et les compétences du porteur de projet. Il permet aussi d'éviter autrement le risque de fraudes.

Concernant le processus d'évaluation même du projet, celui-ci peut prendre différentes modalités selon les plateformes. Certaines plateformes ont choisi d'adopter un processus traditionnel similaire à celui de l'investissement en private equity ou en crédit bancaire classique. C'est le cas des plateformes de prêts pour lesquelles des garanties peuvent parfois être demandées, en plus d'un historique comptable de l'entreprise. Ce type de plateformes semble relativement peu approprié pour des jeunes entreprises innovantes, ces dernières ne pouvant présenter les garanties exigées (hypothèques, nantissement de matériels importants, etc.) pour couvrir les différents risques, en plus de ne pouvoir afficher un historique comptable ou financier suffisant. Des plateformes ont mis en place un processus de présélection, soit par un jury interne, soit par un comité composé de membres de la plateforme et de personnalités extérieures (anciens porteurs de projets, experts sectoriels, etc.). D'autres plateformes vont plus loin en faisant valider cette présélection par un $e$-vote des internautescontributeurs. Selon les déclarations d'un responsable de plateforme, "ce mécanisme permet un dialogue entre le porteur de projet et la foule ». Il s'avère "également être créateur de confiance dans le sens où il permet de construire un avis stable et motivé par la foule sur la réussite du projet ». Le e-vote permet à la plateforme de combler les déconnections à l'intérieur des réseaux sociaux en reliant les membres de la communauté de contributeurs entre eux, mais aussi avec le porteur de projet ; le e-vote permet également d'assurer un rôle d'intermédiaire informationnel permettant de résoudre les problèmes de sélection adverse (choix de mauvais projets à la place de bons projets, et inversement). Selon Agrawal et al. 
(2015), le e-vote permet aussi de réduire les coûts liés à la dispersion géographique des membres de la communauté de contributeurs dans l'espace mondial (coûts des asymétries d'information sur les projets externes à son territoire ; coûts liés à la due diligence réalisée « à distance »). Certaines plateformes ont mis en place un modèle dit de triangulation. Ce modèle s'appuie sur le recours à l'expertise d'acteurs externes tels que les experts-comptables, les capital-risqueurs et autres Business Angels. C'est l'exemple de la platerforme Anaxago ayant initié un partenariat avec l'Ordre des experts-comptables pour "promouvoir cette nouvelle forme de financement tout en la sécurisant : l'expert-comptable atteste en amont les informations financières prévisionnelles des projets proposés ». Un autre exemple est celui de la plateforme Sowefund qui ne propose à ses investisseurs que des projets déjà investis par des réseaux de Business Angels. D'autres PC ont mis en place un principe de « syndicate » ou co-investissement (Deffains-Crapsky et al., 2016), qui permet à la foule de contributeurs individuels d'investir aux côtés d'investisseurs professionnels. Ainsi, le principe mis en place par Sowefund constitue clairement un mécanisme de co-investissement entre la foule et les professionnels du capital-risque au sens large. Des réseaux de Business Angels, tel IT Angels ou VAI Angels, investissent dans les projets sélectionnés, puis présentés sur la plateforme aux côtés de contributeurs individuels. Un partenariat initié entre Sowefund et le Syndicat FundMe s'inscrit également dans cette logique. Le principe permet notamment de faciliter la collaboration entre les investisseurs novices et les investisseurs experts. A noter que certaines plateformes s'abstiennent d'organiser une sélection par le e-vote, en préférant organiser une sélection en interne pour éviter le risque de fraudes, et notamment les manipulations par les porteurs de projets qui peuvent très bien inviter leur entourage à émettre des commentaires favorables.

Enfin, à côté des dispositifs de réduction des risques existants au sein des PC, le développement du crowdfunding a également conduit le législateur à mettre en place un cadre juridique dédié à la prévention des risques.

\section{Les dispositions juridiques de prévention des risques}

La règlementation a mis en place certains mécanismes visant à prévenir la réalisation du risque, tant au niveau de l'exercice de l'activité par les plateformes, qu'au niveau de la perte en capital et à celui du défaut de remboursement des fonds engagés par les contributeurs. Lorsque ce risque se réalise, il existe également des mécanismes juridiques visant à engager la responsabilité de la PC ou celle du porteur de projet.

\section{Les mécanismes visant à prévenir la réalisation du risque}

Les mesures de prévention sont organisées par des mécanismes issus du droit bancaire et financier. Dans ce cadre, le régime juridique se fonde actuellement sur la délivrance par l'Autorité des marchés financiers (AMF) et/ou par l'Autorité de contrôle prudentiel et de résolution (ACPR) d'un agrément aux plateformes. La règlementation opère une sélection en proposant aux plateformes qui souhaitent réaliser des opérations de souscription de titres financiers le choix entre deux statuts : le statut de Conseiller en Investissement Participatif (CIP) et celui de Prestataire en Service d'Investissement (PSI).

Le statut de CIP est accessible sans contrainte capitalistique mais il est réservé aux personnes morales établies en France, et exerçant à titre de profession habituelle une activité exclusive de conseil en investissement portant sur des offres de titres financiers au moyen d'un site Internet. L'accès à ce statut est subordonné à l'immatriculation au registre des intermédiaires 
(ORIAS) et à l'adhésion à une association professionnelle agréée par l'Autorité des marchés financiers (AMF). Les personnes physiques qui administrent les CIP sont soumises à des exigences d'honorabilité et de compétences professionnelles par l'article L. 547-3 du CMF (elles ne peuvent par exemple pas être sous le coup d'une sanction de l'AMF ou avoir été condamnées pour crime, fraude fiscale, ...). Les CIP doivent également souscrire une assurance de responsabilité civile professionnelle et mettre en place des dispositifs de conformité. Ils ne peuvent pas offrir plus de 2,5 millions d'euros de titres financiers par période de 12 mois. Les PSI quant à eux sont, selon l'AMF, des «entreprises d'investissement et des établissements de crédit ayant reçu un agrément pour fournir des services d'investissement. L'exercice de chacun de ces services est soumis au respect de règles de bonne conduite et d'organisation ; après avis de l'AMF, l'agrément est délivré par l'Autorité de contrôle prudentiel et de résolution (ACPR) qui tient à jour la liste des prestataires agréés ». Les PSI doivent également souscrire à une assurance de responsabilité civile professionnelle. Les contraintes capitalistiques du PSI sont importantes puisque son agrément est subordonné à la constitution d'un capital minimum de 50000 euros, s'il ne détient pas de fonds appartenant à la clientèle, et de 125000 euros minimum s'il en détient.

Par ailleurs, le CIP ne peut conserver les fonds, ni détenir les titres (actions, obligations, etc.) de la clientèle. Cette interdiction va permettre une limitation du risque de perte des fonds en cas de défaut de la plateforme. En revanche, cette interdiction ne concerne pas le statut de PSI.

La règlementation (Code monétaire et financier) soumet les plateformes à une obligation d'information et de vigilance. Les plateformes doivent fournir à l'investisseur les renseignements portant sur les modalités de l'opération, ainsi que ceux nécessaires à l'appréciation du projet d'investissement (derniers comptes de l'émetteur, droits attachés aux titres offerts...). Les CIP et les PSI doivent également avoir un site internet "progressif », l'objectif étant de faire prendre conscience à l'investisseur des risques potentiels. Une première étape permet de s'assurer que l'internaute comprend la nature de tout projet d'investissement ainsi que les risques associés. Il ne pourra accéder au détail des offres qu'après avoir pris connaissance et accepté les risques auxquels il s'engage et répondu à une liste de questions dont les réponses peuvent orienter la plateforme dans le choix d'accepter ou non l'accès à ses offres. La seconde étape, réalisée avant la souscription, consiste pour la plateforme à faire passer un test d'adéquation visant à s'assurer que l'offre correspond à l'expérience, aux connaissances ainsi qu'à la situation familiale et patrimoniale de l'internaute. Il en résulte que les plateformes doivent s'enquérir auprès des contributeurs potentiels de leur connaissance et expérience en matière d'investissement, ainsi que de leur situation financière et des objectifs poursuivis afin de leur proposer un placement adapté à leur situation.

Les plateformes sont également soumises à une obligation de vigilance. Les articles L. 547-9 et L. 548-6 du Code monétaire et financier imposent aux CIP et aux PSI une obligation d'information sur les risques auxquels les contributeurs s'exposent. L'AMF impose d'ailleurs que ces derniers ne puissent accéder aux offres qu'après avoir consulté un avertissement de sensibilisation et répondu à un questionnaire avant de pouvoir accéder à des informations plus détaillées sur les projets. Par ailleurs, les CIP ont l'obligation de mettre en place une politique de gestion des conflits d'intérêts pouvant nuire aux intérêts des clients.

La règlementation a également créé le statut d'intermédiaire en financement participatif (IFP) pour les plateformes qui jouent un rôle d'intermédiaire dans la souscription de prêts. L'article 
L. 548-2 du Code monétaire et financier (CMF) définit l'activité des IFP d'une part comme « les personnes qui exercent, à titre habituel, l'intermédiation au sens de l'article L. 548-1 pour les opérations de prêt à titre onéreux ou sans intérêt », et d'autre part, comme "les personnes qui exercent, à titre habituel, l'intermédiation au sens de l'article L. 548-1 et qui ne proposent que des opérations de dons ». L'activité des IFP est soumise à certaines limites puisqu'ils ne peuvent exercer que des activités mentionnées à l'article L. 548-1 du CMF ou, le cas échéant, celles qu'ils sont autorisés à exercer habituellement. L'IFP est considéré comme un opérateur de plateformes en ligne, tel que défini par l'article L. 111-7 du Code de la consommation. Il en résulte pour l'intermédiaire de nouvelles obligations d'information et de loyauté qui viennent s'ajouter à celles liées à leur statut particulier. Le dirigeant de l'IFP doit remplir des conditions d'honorabilité, de compétences professionnelles et souscrire à une assurance en responsabilité professionnelle. A ce titre les IFP doivent également s'immatriculer sur le registre unique tenu par l'ORIAS (art. L.546-1-I et art. L.548-3 du Code monétaire et financier) et souscrire une assurance en responsabilité civile professionnelle. Le décret du 28 octobre 2016 a également fixé un plafond s'agissant de la souscription de prêts par les contributeurs afin de limiter les risques : 2000 euros pour le prêt avec intérêt et 5000 euros pour le prêt sans intérêt.

Ces mécanismes ne font pas disparaitre le risque de défaut de paiement des emprunteurs, mais permettent toutefois de les limiter. Au demeurant, se pose aussi la question de la responsabilité des porteurs de projets en cas de faillite, mais aussi celle des PC organisatrices de l'opération.

\section{Les mécanismes classiques de responsabilité en cas de réalisation du risque}

Lorsqu'un contributeur subit un préjudice dans le cadre d'un investissement en capital, il peut envisager de mettre en jeu la responsabilité du porteur de projet qui a ouvert son capital en proposant des actions ou celle de la plateforme ayant joué le rôle d'intermédiaire.

\section{-La responsabilité du porteur de projet}

Les contributeurs sont exposés à trois risques principaux en cas de défaillance de la société financée : celui de l'illiquidité des actions, d'un rendement moins important que celui espéré ou d'une perte de tout ou partie des capitaux investis.

L'ordonnance $\mathrm{n}^{\circ}$ 2014-559 du 30 mai 2014 a facilité le recours au crowdfunding par souscription d'actions. Deux formes de statuts juridiques peuvent proposer leurs titres au public : la société anonyme (SA) et la société par actions simplifiées (SAS). Les investisseurs qui apportent des fonds à une SA ou à une SAS en passant par une PC deviennent des actionnaires en contrepartie de ce qui constitue un apport en numéraire. En cas de défaillance de la société, il ne sera pas possible d'agir contre ses actionnaires. Les sociétés par actions permettent en effet aux détenteurs d'actions de bénéficier d'une limitation de leur responsabilité à hauteur des apports réalisés en contrepartie du risque pris à perdre les capitaux investis. Les actionnaires n'ont pas d'obligations à la dette sociale (les créanciers de la société ne peuvent agir contre eux directement). De même, si la société subit des pertes, les actionnaires ne seront pas tenus d'y contribuer au-delà de leurs apports (la société sera donc mise en cessation de paiement si l'actif social ne permet pas de couvrir le passif). Si cette défaillance est frauduleuse, on pourrait imaginer une mise en jeu de la responsabilité personnelle des dirigeants en cas de faute prouvée mais cette hypothèse reste rare. En cas d'illiquidité ou de rendement moins important que celui annoncé, il sera possible d'envisager 
de mettre en jeu la responsabilité de la société. Cette responsabilité sera de nature contractuelle (les apporteurs en capitaux ayant conclu un contrat de société). Il sera donc nécessaire d'établir une faute de la société, un préjudice ainsi qu'un lien entre ces éléments. La faute semble difficile à établir car tout investissement dans le capital d'une entreprise comporte en soi un risque. Par ailleurs, les mentions de sensibilisation aux risques imposées aux plateformes semblent s'opposer à ce qu'un investisseur puisse agir en cas de perte de valeur de l'action ou de difficulté à la céder.

Malgré tout, le contributeur peut toujours agir contre le porteur de projet par une action de groupe, encadrée par la loi Hamon n²014-344 du 17 mars 2014. Cette action prévoit que des associations représentatives peuvent intenter une action en justice pour la réparation de préjudices patrimoniaux subis par des consommateurs. Or, l'investisseur qui souscrit via Internet des titres financiers est considéré comme un consommateur de services financiers (article 1 de l'Ordonnance 2005-648 du 6 juin 2005 ayant modifié l'article L. 121-20-8 du Code de la consommation). Ainsi, «par l'intermédiaire d'une association de défense des consommateurs représentative au niveau national et agréée pour agir devant une juridiction civile, un contributeur pourra engager la responsabilité d'un porteur de projet s'il démontre qu'il a commis un manquement à ses obligations légales ou contractuelles » (Touati, 2016).

Le contributeur peut également saisir l'AMF pour engager une procédure de médiation gratuite, mais qui n'a pas de force contraignante, en veillant toutefois au fait que cette «médiation ait été précédée d'une première démarche écrite ayant fait l'objet d'un rejet total ou partiel auprès du PSI» ou de l'émetteur du projet concerné.

\section{- La responsabilité de la plateforme}

La règlementation spécifique au crowdfunding n'a pas, de façon assez surprenante, abordé la question de la responsabilité des plateformes. Il faut toutefois relever la possibilité pour un contributeur d'engager la responsabilité pénale de la plateforme en tant qu'hébergeur sur le fondement de l'article 6, de la Loi pour la confiance en l'Économie numérique du 21 juin 2004. Celle-ci prévoit que «la responsabilité pénale pourra être mise en æuvre si l'hébergeur a connaissance de contenus présentant un caractère manifestement illicite et qu'il n'a pas agi promptement pour les retirer dès qu'il en a eu connaissance ».

En dehors de ce cas particulier, la responsabilité civile d'une plateforme pourrait être recherchée sur le fondement du régime de responsabilité professionnelle autonome pesant sur les IFP et les CIP (Touati, 2016) (C

$f$. obligation d'information et obligation de vigilance). En cela, il est possible de se référer au droit commun de la responsabilité contractuelle (il y a un contrat de prestation de services entre la plateforme et l'investisseur). La mise en œuvre de la responsabilité de la plateforme suppose donc la réunion du triptyque vu précédemment (faute, dommage et lien de causalité). S'agissant de la faute, il pourrait s'agir d'un manquement à des obligations pré-contractuelles (information ou vigilance notamment) ou contractuelles. Cette faute sera simple à établir en cas de manquement de la plateforme à une obligation légale (hypothèse qui devrait être rare) mais plus complexe lorsque la plateforme s'est conformée à ses obligations légales mais que l'investissement s'est avéré hasardeux. Un investisseur ayant subi des pertes financières devra établir que la plateforme a commis une faute. Le contentieux devrait porter sur l'obligation d'information et de conseil. En la matière, les plateformes ont une obligation de moyens. S'agissant du préjudice, celui-ci pourra être de façon classique une perte en capital. Se pose la question de la perte de chance qui est indemnisée par les juges (Chaaben, 2016, p. 90). Le 
préjudice pourra ainsi être constitué de la perte de chance de ne pas avoir pu éviter ou limiter les pertes. La Cour de cassation a ainsi jugé responsable une société d'investissement ayant privé sa cliente de la chance d'avoir pu éviter un risque s'étant finalement réalisé, par une décision plus judicieuse, et ayant entrainé une perte, laquelle constitue un préjudice distinct de celui résultant des opérations qu'elle a effectivement réalisées ou fait réaliser (Cass. com., 10 décembre 1996, n94-16 082). Le plus complexe sera sans doute de prouver le lien de causalité. La causalité dépend en effet, à la fois d'éléments de fait, tels que l'ampleur de la faute de la plateforme, ou des circonstances dans lesquelles le préjudice est survenu ou encore de la qualité de l'investisseur. Dès lors, si l'investisseur ne démontre pas que le dommage résulte de la faute de la plateforme, alors, l'action en responsabilité sera rejetée par les juges.

En définitive, il semble nécessaire que les parties prenantes anticipent et gèrent les risques en se servant notamment du contrat. Dane ce cadre, les clauses contractuelles peuvent permettre de limiter le risque, de le mettre à la charge d'une partie ou de le reporter sur un tiers. Les plateformes ont ainsi souvent recours à des clauses limitatives ou exonératoires de responsabilité dans leurs conditions générales. Ces clauses, qui pourraient être prohibées si l'investisseur est un consommateur, sont en revanche valables si l'investisseur est un professionnel, sauf à considérer que le contrat conclu avec la plateforme est un contrat d'adhésion. Cette distinction a été introduite par la réforme récente du droit des contrats dans l'Ordonnance du 10 février 2016. Le contrat sera qualifié de contrat d'adhésion lorsque «les conditions générales, soustraites à la négociation, sont déterminées à l'avance par l'une des parties » (article 1110 du Code civil). On retrouve dans les contrats conclus avec les plateformes la présence des trois éléments: les conditions générales, la détermination unilatérale et à l'avance desdits conditions générales par l'une des parties au contrat, et l'absence de négociation portant sur ces conditions générales. Il pourra être possible d'appliquer dans cette situation le nouvel article 1171 du Code civil rendant invalides les clauses abusives dans les contrats d'adhésion (en cas de déséquilibre significatif des droits et des obligations des parties au détriment de l'adhérent). Ces dispositions rendent donc plus complexe la possibilité pour les plateformes de limiter ou de s'exonérer de leur responsabilité. Ces clauses pourront toutefois être insérées afin qu'elles aient un impact psychologique sur un contractant qui ignorerait ces dispositions légales et donc la possibilité de contester ces stipulations en justice.

Le risque peut également être reporté sur un tiers. Une plateforme pourra ainsi proposer au contributeur de souscrire un contrat d'assurance couvrant certains risques et bénéficier d'une option de vente de ses titres à un fonds d'investissement partenaire de la plateforme en cas de réalisation d'un risque (Vabres, 2016, p. 212). Le risque peut également être géré par le biais du contrat de société entre actionnaires (pacte d'actionnaires). Les investisseurs peuvent ainsi bénéficier de clauses de sortie ou de rachat des titres à prix fixe, consenties par les anciens actionnaires ayant souhaité ouvrir le capital de la société afin d'éviter le risque de perte de valeur.

Sur un plan pratique, la principale difficulté pouvant apparaitre est celle liée au caractère limité, voire très faible du préjudice des contributeurs qui n'ont pu investir qu'une centaine d'euros. L'action de groupe peut s'avérer être un outil efficace dans ce cadre. En effet, les contributeurs mécontents pourront potentiellement déposer un recours collectif (Assadi, 2015, p. 144) pour mauvaise exécution du contrat. Le juge statuera alors sur la recevabilité de l'action et définira le groupe de consommateurs pouvant faire partie de l'action. 


\section{CONCLUSION}

Dans cet article, nous avons souhaité appréhender les enjeux managériaux et juridiques du risque dans le financement de projets innovants, à partir d'une approche bi-disciplinaire à la croisée des sciences de gestion et du droit. Nous avons focalisé notre travail sur le crowdfunding basé sur le rendement financier (CBRF) car se prêtant parfaitement à une démarche d'appréhension du risque et par ailleurs soumis au cadre de la réglementation bancaire et financière. Le développement du crowdfunding est un mouvement de fond dont l'essor ne fait que commencer (Bessière, Stéphany 2017, p. 155). Aussi, en facilitant les échanges directs entre les porteurs de projets et les investisseurs-contributeurs, les plateformes de crowdfunding (PC) se positionnent aujourd'hui comme un nouveau modèle d'intermédiation financière chargé de collecter l'information en vue de réduire les risques pour l'investisseur (Dardour, 2015). Ces risques sont potentiellement nombreux ; certains correspondent à des risques classiquement identifiés dans les circuits de financement traditionnels ; d'autres, plus spécifiques sont inhérents au caractère en ligne de l'activité. L'article a également permis de présenter les solutions réglementaires mises en œuvre par les autorités de tutelle en vue de protéger l'épargne des contributeurs. Les résultats de l'article peuvent offrir des informations intéressantes non seulement pour les investisseurs et les porteurs de projets, mais aussi pour les organismes régulateurs. L'émergence du crowdfunding semble notamment avoir introduit et/ou accentué une caractérisation distincte de deux groupes d'investisseurs sur les marchés de capitaux, à savoir les investisseurs traditionnels qui sont principalement les investisseurs professionnels (Business Angels, capital-risqueurs, fonds et banques d'investissement, etc.) et un autre groupe constitué par la «foule» de petits investisseurs sur internet. Certes, l'hétérogénéité dans la composition de la foule de contributeurs est une caractéristique essentielle (Bessière, Stéphany, 2017, p. 141). Le développement de celle-ci devant permettre notamment de promouvoir une culture du coinvestissement (syndication) (Deffains-Crapsky et al., 2016) à même de permettre une réduction de l'exposition au risque, mais également une meilleure sélection des projets par un partage des expertises. Elle devrait également permettre d'apporter des réponses aux problèmes de financement que rencontrent les jeunes entreprises innovantes. Cependant, le fait que les petits investisseurs soient susceptibles de manquer d'expertise financière par rapport aux investisseurs professionnels (Freear et al., 1994) implique, selon nous, l'instauration de conditions équitables dans les procédures de levée de fonds. Or à ce jour, le sujet n'est pas encore abordé dans les textes réglementaires en vigueur. Le crowdfunding pourrait s'inspirer des pratiques sur les marchés financiers pour équilibrer les règles du jeu. Par exemple, dans le cadre des introductions en bourse, l'autorité des marchés financiers (AMF) a instauré l'obligation de prévoir une tranche spéciale à destination des investisseurs particuliers ${ }^{8}$. Les procédures d'introduction en bourse ont ainsi été conduites à se structurer schématiquement en deux tranches : une tranche ouverte au public de particuliers et une tranche dédiée aux investisseurs institutionnels. Cette disposition permet d'affaiblir toute tentative d'arbitrage des plus gros investisseurs qui voudraient réaliser des profits au détriment des petits porteurs. Ainsi, si toutes les catégories de contributeurs bénéficient d'un minimum d'égalité de traitement, le crowdfunding pourra alors être une option de financement alternative et un produit d'investissement plus durable.

Les résultats de l'article font également progresser les connaissances actuelles et encore limitées sur le risque en crowdfunding. Cela étant, nous sommes toujours dans une phase

\footnotetext{
${ }^{8}$ Qui résulte de l'article 315-355 du règlement général de l'AMF - Titre III - Prestataires de services d'investissement - Chapitre V « Autres dispositions »- Section 2 « Gestion des informations privilégiées et restrictions applicables au sein des prestataires de services d'investissement ».
} 
d'apprentissage d'un marché dont on ne connait pas encore tous les ressorts. Certes, le crowdfunding permet à de nombreux porteurs de projets de récolter des fonds. Mais combien de ces projets deviendront des activités pérennes, viables et créatrices de valeur ajoutée ? De cette question découle l'une des principales limites de cet article. En effet, si l'analyse développée fait progresser les connaissances sur le risque en crowdfunding, celle-ci se limite à la compréhension des processus de traitement et de prévention de ces risques. Nous n'avons donc aucun élément d'appréciation concernant la réussite future des projets financés. Or le crowdfunding n'échappe pas à cette règle bien connue en affaires : tout projet d'investissement comporte des incertitudes. Savoir si les projets ayant réussi à franchir le filtre des plateformes et à mobiliser avec succès les contributeurs sont également ceux dont la rentabilité des investissements et la pérennité sur le long-terme sont assurées, devrait faire l'objet de recherches futures. Il s'agit clairement d'une tâche difficile aujourd'hui pour les chercheurs, compte tenu de la jeunesse du secteur d'activité. Aussi, à l'avenir, il conviendrait également de suivre l'évolution de la croissance du marché, car celle-ci déterminera, le cas échéant, les ajustements règlementaires nécessaires ou l'élaboration de nouveaux textes législatifs. En tout état de cause, toute nouvelle règlementation ou ajustement devra être élaboré de manière flexible et allégé, de sorte à ne pas brider l'innovation, et afin que les futures innovations du crowdfunding puissent se développer au sein d'un cadre normatif adapté.

\section{BIBLIOGRAPHIE}

AGRAWAL, A., CATALINI, C., GOLDFARB, A. (2015), Crowdfunding: Geography, Social Networks, and the Timing of Investment Decisions, Journal of Economics and Management Strategy, 24(2), 253-274.

AGRAWAL, A. K., CATALINI, C., GOLDFARB, A. (2013), Some Simple Economics of Crowdfunding, Innovation Policy and the Economy, 14, 63-97.

ASSADI, D. (2015), Strategic approaches to successful crowdfunding, IGI Global, 1ère Edition, 154-163.

BESSIÈRE, V., STÉPHANY, E. (2017), Le crowdfunding. Fondements et pratiques, $2^{\text {ème }}$ Edition, Paris, DeBoeck Supérieur.

CHAABEN, M. (2016), Le financement participatif, Saint-Denis, Connaissances et savoir.

COFACE (2015), La France, une terre propice au développement des Start-Ups? Risque pays et études économiques, Panorama, Les publications économiques de COFACE.

CORNELL, C. J., LUZAR, C. (2014), Crowdfunding Frad: How Big is the Threat?, Crowdfund Insider (https://www.crowdfundinsider.com/2014/03/34255-crowdfunding-fraudbig-threat/).

DARDOUR, A. (2015), La gestion du risque par les opérateurs de crowdfunding d'entreprise, Recherches en Sciences de Gestion, 107, 45-65.

DEFFAINS-CRAPSKY, C., CUENOUD, T., GLEMAIN, P. (2016), Les déterminants de la syndication avec les Business Angels dans les opérations d'Equity Crowdfunding : le cas français, Document de travail du GRANEM n ${ }^{\circ}$ 2016-01-047.

EISENHARDT, K. (1989), Building Theories from Case Study Research, The Academy of Management Review, 14(4), 532-550.

FINANCEMENT PARTICIPATIF FRANCE (FPF) (2017), Répertoire des plateformes membres de FPF (http://financeparticipative.org/repertoire-des-plateformes-membres-de-fpf/). FORTIN, M. (1996), Le processus de la recherche, de la conception à la réalisation, Ville Mont-Royal, Québec, Décarie éditeur. 
FREEAR, J., SOHL, J. E., WETZEL, W. E. (1994), Angels and non-angels: are there differences?, Journal of Business venturing, 9(2), 109-123.

KIRBY, E., WORNER, S. (2014), Crowd-funding: An Infant Industry Growing Fast, Staff Working Paper of the IOSCO Research Department, SWP 2014/3, Madrid, OICV-IOSCO.

MAHADEVAN, B. (2000), Business Models for Internet-Based E-Commerce: An Anatomy, California Management Review, 42(4), 55-69.

MILES, M., HUBERMAN, M. (2003), Analyse des données qualitatives, Paris, De Boeck. ONNEE, S. (2016), Vers une compréhension du rôle joué par la foule, Réalités Industrielles : une série des Annales des mines, 2016/1, 12-16.

QUAGLIA, L. (2010), Governing Financial Services in the European Union, London, Routledge.

TOUATI, A. (2016), La responsabilité des plateformes de crowdfunding : regards croisés sur les enjeux du financement participatif, Les Echos.fr, 21/03/2016 (https://www.lesechos.fr/idees-debats/cercle/cercle-155414-la-responsabilite-des-plateformesde-crowdfunding-regards-croises-sur-les-enjeux-du-financement-participatif-1208619.php).

VERNIMMEN, P., QUIRY, P., LE FUR, Y. (2017), Finance d'entreprise, Paris, Dalloz, $12^{\text {ème }}$ Edition.

VABRES, R. (2016), Le financement participatif : Quel outil d'investissement? Quelle protection des investisseurs ?, Revue de jurisprudence commerciale, 3, 204-212.

YIN, R (1994), Case study research. Design and methods, London, Sage Publications. 